\title{
MMPs and TIMPs expression in facial tissue of children with cleft lip and palate
}

\author{
Liene Smane-Filipovaa ${ }^{a}$ Mara Pilmane ${ }^{a}$, Ilze Akota ${ }^{b}$
}

\begin{abstract}
Background and Aims. Morphogenesis of the upper lip and palate is a complex process involving highly regulated interactions between epithelial and mesenchymal cells. Genetic evidence in humans and mice indicates the involvement of matrix metalloproteinases (MMPs) and their endogenous tissue inhibitors (TIMPs) in cleft lip palate (CLP) aetiology. This study investigated whether expression of MMP-2, MMP-8, MMP-9, TIMP-2, and TIMP-4, which are essential for the upper lip and palate fusion, is dysregulated in children with CLP.

Methods. Oral mucosa tissue samples were obtained from patients with complete unilateral (CU) CLP $(n=25)$ and complete bilateral (CB) CLP $(n=19)$ during corrective plastic surgery and in unaffected control subjects $(n=10)$. MMPs and TIMPs expression was assessed by immunohistochemistry, and the data were analyzed using the Kruskal - Wallis test with the Bonferroni correction.

Results. In CLP patients, MMP-2, TIMP-2 immunoreactivity in the oral mucosa was seen to have a few to abundant structures, but the overall number of MMP-2, TIMP-2-positive structures was greater than that in controls $(P<0.01)$. The total number of TIMP-4, MMP-9-positive cells showed a significant decrease in the CBCLP compared with that of CUCLP $(P<0.001)$. MMP-8 expression trends in the CLP group were similar to those of the control group.

Conclusions. The results suggest that TIMP-4 and MMP-9 are the main ECM remodeling regulatory proteins expressed in CUCLP affected tissues of the oral mucosa. The increased expression of MMP-2 and TIMP-2 in CLP tissues implicates these factors in the regulation of cell migration during ECM turnover independently of different types of clefts. Investigation of MMP and TIMP expression in tissue samples from patients with CLP appears to be a promising approach to the etiopathogenesis of CLP.
\end{abstract}

Key words: cleft lip and palate, matrix metalloproteinases, tissue inhibitors of matrix metalloproteinases

Received: June 6, 2016; Accepted: October 24, 2016; Available online: November 21, 2016 https://doi.org/10.5507/bp.2016.055

${ }^{a}$ Department of Morphology, Institute of Anatomy and Anthropology, Riga Stradins University, Dzirciema Street 16, Riga LV 1007, Latvia 'Institute of Stomatology, Riga Stradins University, Riga, Latvia

Corresponding author: Liene Smane, e-mail:Liene.Smane@rsu.Iv

\section{INTRODUCTION}

Craniofacial anomalies, in particular cleft lip and palate (CLP), are the most prevalent birth defects in humans with a worldwide frequency of 1 in 500 to 1000 livebirths ${ }^{1}$. About $70 \%$ of cases are considered non-syndromic CLP (NSCLP), whereas the remaining approximately $30 \%$ of CLP are syndromic ${ }^{2}$. Clinically, cleft lip and palate is a unilateral or bilateral gap between the philtrum and the lateral upper lip.

The etiology of this pathology has only been partially explained to date. In both humans and mice, it is known that environmental and epigenetic factors affect NSCLP susceptibility ${ }^{3}$. However, in most non-syndromic cases, the cause remains unknown. CLP is characterized by the incomplete fusion of the lip, alveolus, and hard and soft palates, with the most severe clefts extending completely through both soft tissue and bone. The mechanisms for explaining the incomplete fusion of the facial structures are complex and not fully understood. Three main mechanisms have been proposed: programmed cell death ${ }^{4}$, cell migration to the oral and nasal side of the palate ${ }^{5}$, and epithelial-mesenchymal transdifferentiation ${ }^{6}$. Normal development of the lip and palate involves a series of highly coordinated morphogenetic events that include cell migra- tion, differentiation, growth, apoptosis, active fusion, and subsequent breakdown of the medial edge epithelium, as well as breakdown of the basement membrane. Any disturbance in these events may cause irreversible changes resulting in the development of facial clefts.

Some proteases, such as matrix metalloproteinases (MMPs), are responsible for remodeling of the extracellular matrix (ECM) and mediate changes in the basement membrane components of the medial edge seam during lip and palate fusion and their imbalance can lead to CLP $\left(\right.$ ref. $\left.^{7}\right)$. Several studies demonstrate that tissue remodeling by MMPs plays an important role in the late stages of lip and palate formation. Together with their endogenous tissue inhibitors (TIMPs) MMPs are generally rarely expressed in healthy tissues. Their expression increases during physiological or pathological tissue remodeling with the influence of signaling molecules able to modulate gene expression ${ }^{8}$. MMPs and TIMPs have been suggested to be candidate genes based on research of their spatial and temporal expression patterns during palatogenesis in animals 9 .

Thus, the process of CLP morphogenesis is closely accompanied by changes in the composition of the extracellular matrix that promote cell migration and differentiation, cell-cell interactions, and tissue resorption. 
This study investigated the expression of MMP-2, MMP8, MMP-9, TIMP-2, and TIMP-4 in complete unilateral (CU), complete bilateral (CB) CLP to identify possible changes in signaling pathways that could lead to the abberant maxillofacial morphogenesis observed in CLP.

\section{MATERIALS AND METHODS}

\section{Study population}

The study was carried out according to the Declaration of Helsinki, and the protocal was independently reviewed and approved by the local Ethics Committee of Riga Stradins University. Written informed consent was obtained from all parents after the nature of the study had been fully explained.

Samples for the study were retrieved from the Cleft Lip and Palate Centre of the Institute of Stomatology of the Riga Stradins University. Forty-four CLP patients were enrolled. Twenty-five children had unilateral CLP, while 19 children had bilateral CLP. There were 19 boys and 6 girls in the unilateral CLP patients and 14 males and 5 females in the bilateral CLP patients.

In our study the tissue samples are not from the foetal period where the development of the lip and palate takes place but from fully development children aged (3 months and 8 years and 5 months). To attain optimal healing and hence cosmetic and functional results, a small piece of mucosa is routinely removed during the operation from borders of the cleft region. Patient samples were obtained during various surgeries, such as labioplasty, veloplasty and uranoplasty. Samples of oral mucosa were collected in upper labial frenectomy from control subjects ranging in age from 5 years to 7 years.

\section{Tissue sample preparation}

For conventional light microscopy and immunohistochemistry, tissue samples were fixed for one day in Stefanini's fixative for $24 \mathrm{~h}$. After fixation, tissue blocks were embedded in paraffin. Three-micrometer thick sections were cut from each block, affixed onto the glass slides, then de-paraffinized in xylene, rehydrated through graded alcohol solutions and stained with hematoxylineosin.

\section{Immunohistochemistry}

Three-micrometer thick sections were cut from the same blocks and placed on adhesive microscope slides with silane treated surfaces for immunohistochemical analysis. Tissue sections were labeled with the following primary antibodies: mouse anti-MMP-2 at 1:100 (sc53630; Santa Cruz Biotechnology, Inc., Santa Cruz, CA, USA), mouse anti-TIMP-2 at 1:100 (sc-21735; Santa Cruz Biotechnology, Inc., Santa Cruz, CA, USA), rabbit antiMMP-9 at 1:100 (sc-10737; Santa Cruz Biotechnology, Inc., Santa Cruz, CA, USA), and rabbit anti-TIMP-4 at 1:100 (sc-30076; Santa Cruz Biotechnology, Inc., Santa Cruz, CA, USA). The slides were processed as follows: de-paraffinization; rehydrated through graded alcohol solutions; endogenous peroxidase activity was blocked by incubation with kit peroxidase blocking reagent for 10 minutes; the antibodies were diluted with the Antibody Diluent (64211; Abcam, Burlingame, CA, USA); HiDef Detection $^{\mathrm{TM}}$ Amplifier (Mouse \& Rabbit) (954D-31; Sigma-Aldrich, Rocklin, CA, USA) was applied for 10 minutes; HiDef Detection ${ }^{\mathrm{TM}}$ HRP Polymer Detector (954D-32; Sigma-Aldrich, Rocklin, CA, USA) was applied for 10 minutes and then stained with HRP-compatible chromogen for $5 \mathrm{~min}$ and counterstained in hematoxylin. For negative controls the primary antibody was replaced by a diluent.

Images were captured using a DC 300F camera (Leica, Wetzlar, Germany) and the image processing and analysis software Image Pro Plus v.6.0 (Media Cybernetics, Silver Spring, MD, USA). The intensity of immunostaining was graded semi-quantitatively on a scale of 0 to ++++ , as follows: 0 , no positive structures; $0 /+$, occasional positive structures; +, few immunoreactive structures; ++ , a moderate number of immunoreactive structures; +++ , numerous immunoreactive structures; and ++++, an abundance of immunoreactive structures in the visual field ${ }^{10}$.

\section{Statistical analysis}

Statistical analyses were performed using SPSS v.20.0 (IBM Corp., Armonk, NY, USA). Results are expressed as means \pm SD. The Kruskal - Wallis test with Bonferroni correction were used to test significant difference between groups. The significance level was set at $P<0.05$.

\section{RESULTS}

\section{CBCLP}

MMP-2 and MMP-8-positive structures were detected in all CBCLP cases, and the presence of MMP-2, MMP8-positive epithelial cells was a few to abundant (Fig. 1, 2 ). The number of MMP-2-positive connective tissue cells varied from occasional to numerous. Occasional MMP-8-positive connective cells were found in CBCLP specimens. Moderate numbers of immunoreactive structures were also observed in the salivary gland cells. In all CBCLP specimens, a few to moderate of TIMP-2-positive oral mucosa cells were seen. TIMP-2 immunoreactivity was detected in epithelial cells, fibroblasts, and macrophages. Of note, only a few isolated epithelial and connective tissue cells stained positively for MMP-9 and TIMP-4 in CBCLP affected tissue.

\section{CUCLP}

MMP-2-positive structures were present in all soft tissue samples, and immunoreactivity was detected in epithelial cells, fibroblasts, and macrophages. The number of MMP-2-positive epithelial cells varied from occasional to moderate but positive connective tissue cells were seen in a few to numerous positive structures. Furthermore, the number of TIMP-2-positive cells varied from occasional to numerous in all specimens but the presence of TIMP4-positive epithelial cells and connective cells was a few to abundant (Fig. 3). It is notable that the immunoreactivity for MMP-9 in CUCLP affected tissue was found in all 


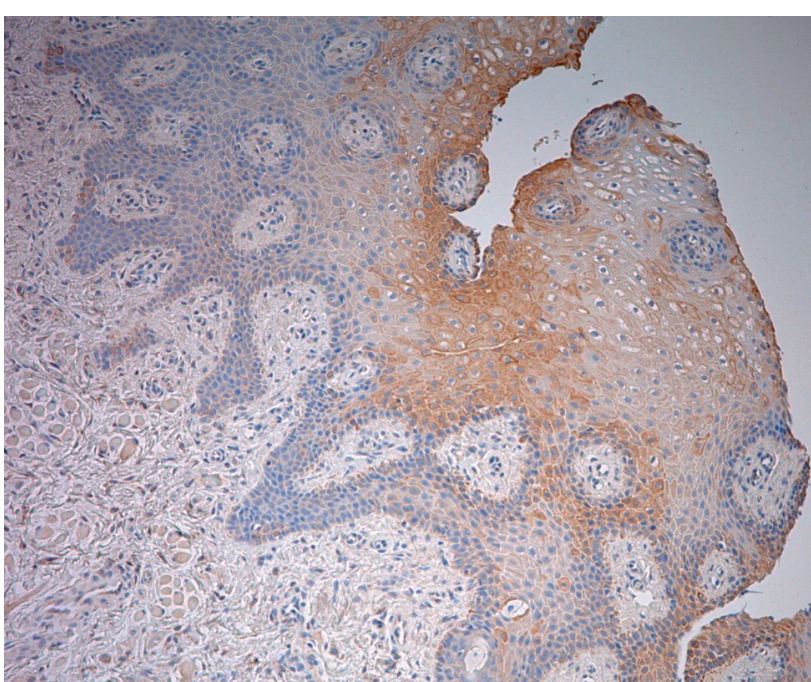

Fig. 1. Moderate MMP-8-positive structures in oral epithelium and connective tissue from the cleft lip tissue of a 3-months-old child. $100 \mathrm{x}$ magnification.

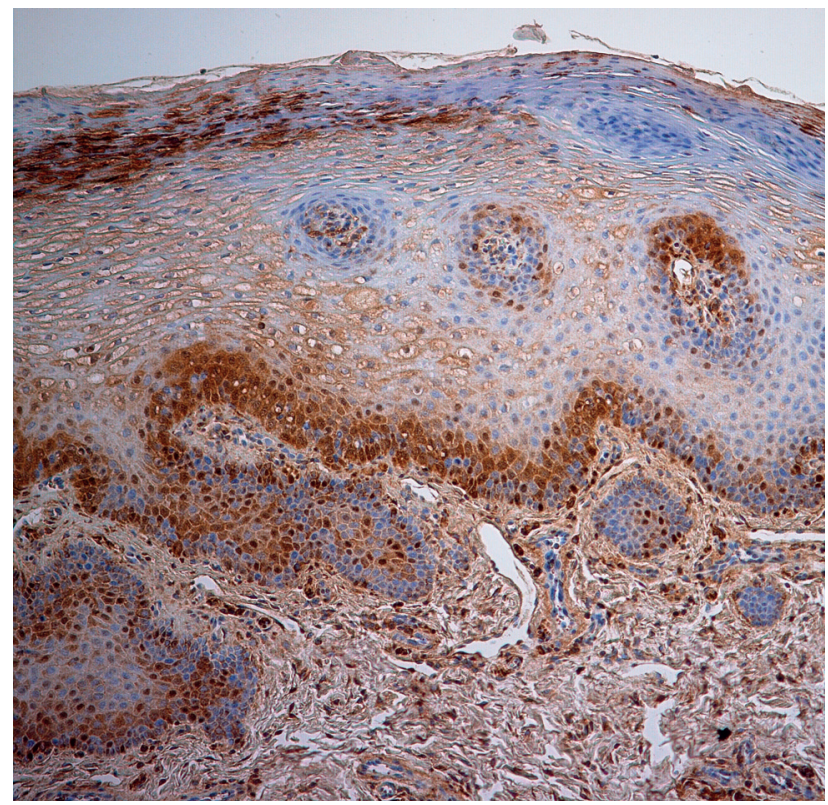

Fig. 3. Abundant TIMP-4-positive cells in basal layer from lip tissue of a 3-months-old child with CUCLP. 100 x magnification.

tissue samples. Occasional to numerous immunoreactive cells were observed in epithelium and connective tissue (Fig. 4, 5).

In all control specimens, only a few of MMP-2-positive epithelial cells were found (Fig. 6). The total number of MMP-2-positive cells was significantly lower in the control than in the CBCLP group $(P<0.016)$. Notably, of the 10 control specimens, 9 were negative for MMP-2 in the connective tissue. There was significant difference between groups in the mean numbers of MMP-2-positive connective cells $(P<0.002 ; P<0.005)$. This aside, the mean number of TIMP-2-positive connective cells was

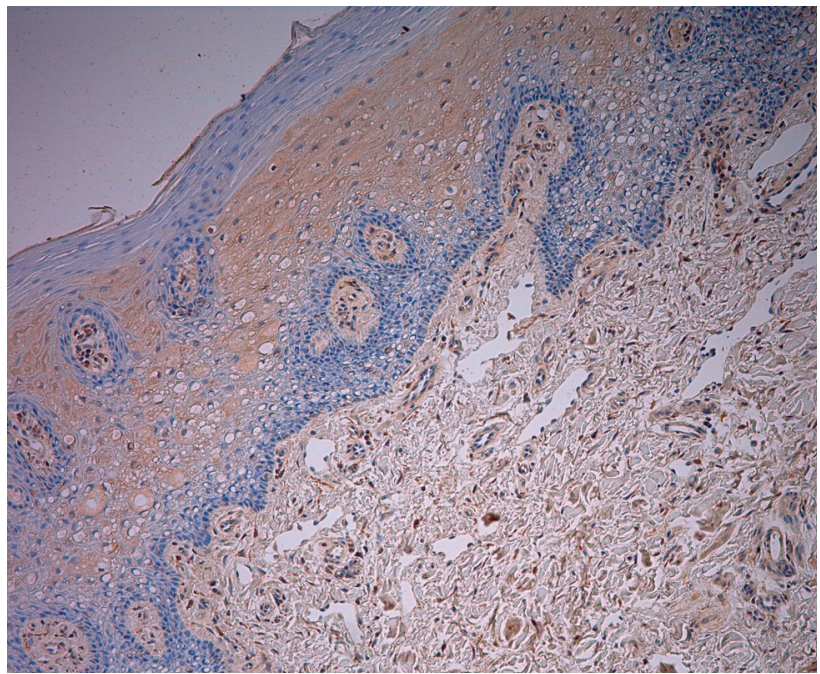

Fig. 2. Abundant MMP-2-positive epithelial cells from lip tissue of 6-moths-old child with CLP. $200 \mathrm{x}$ magnification.

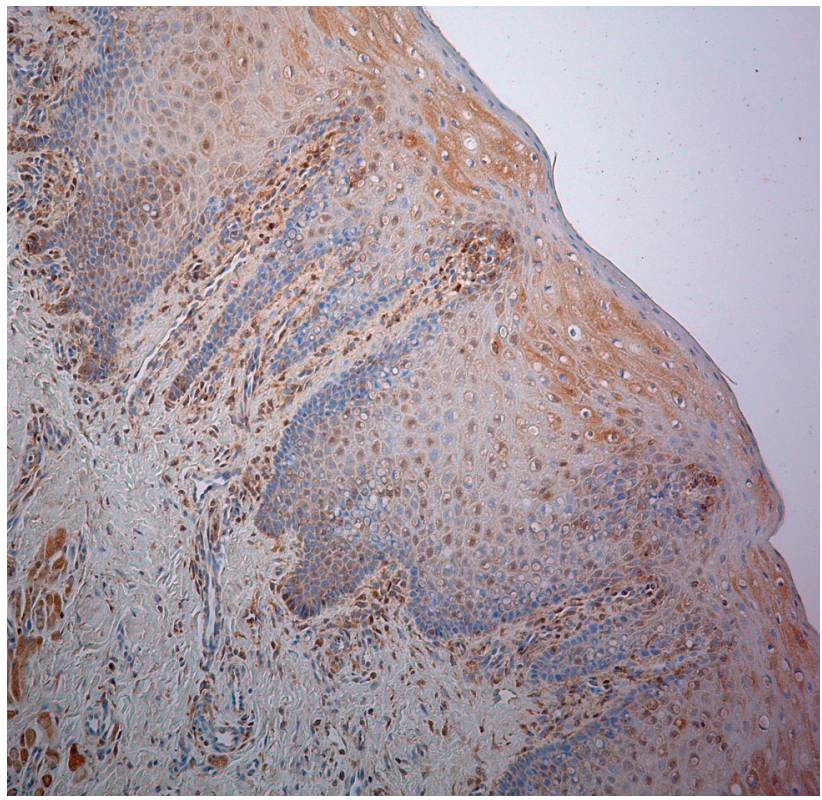

Fig. 4. Moderate MMP-9-positive cells in oral epithelium and connective tissue from the lip tissue of a 4-month-old child with CUCLP. $200 \times$ magnification.

significantly lower in the control group than in the CLP groups $(P<0.005 ; P<0.002)$. Further, the total number of MMP-2-positive cells was significantly lower in the CUCLP than in the CBCLP group $(P<0.001)$. The number of MMP-9-positive cells was significantly higher in the CUCLP than in the CBCLP group and controls ( $P$ $<0.001 ; P<0.005)$. The total number of TIMP-4-positive cells also showed a significant decrease in the CBCLP and controls compared with that of the CUCLP $(P<0.001$; $P<0.002)$. MMP-8 expression trends in the CLP groups were similar to those of the control group. There was no significant difference in mean numbers between groups. 


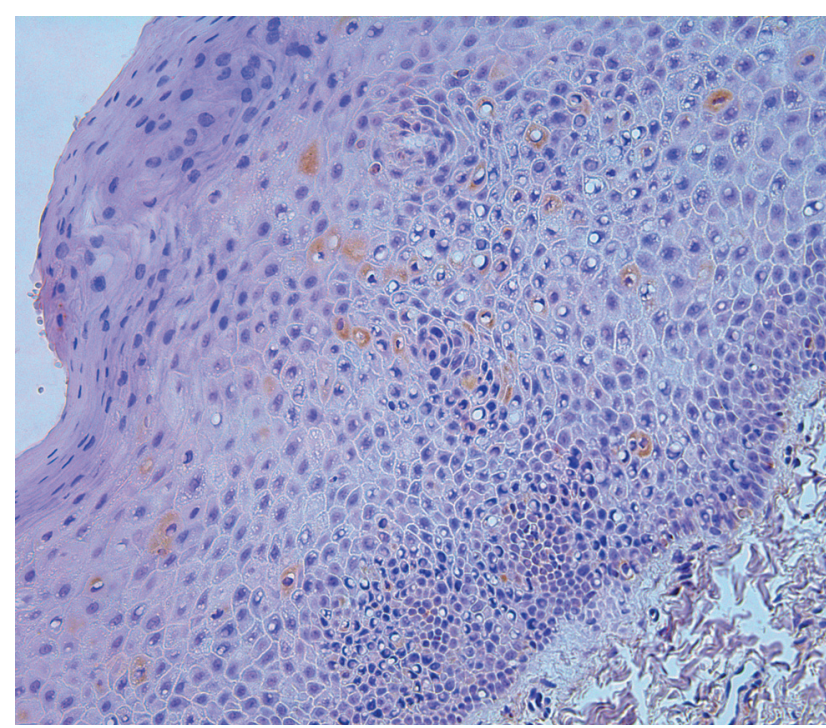

Fig. 5. Sporadic MMP-9 immunoreactivity in oral epithelial cells from the cleft lip tissue of a 4-month-old child with CBCLP. $250 \times$ magnification.

\section{DISCUSSION}

In humans, morphogenesis of the lip and palate fusion is a highly regulated process involving the cooperation of different signalling molecules. The upper lip fusion promotes palate formation, and therefore improper lip fusion may secondarily affect palate fusion ${ }^{11}$. MMPs and TIMPs have the ability to participate in ECM remodelling during lip and palatal fusion and the EMT associated with this process. Controlled remodelling of the ECM is an essential aspect of craniofacial morphogenesis. Experiments in mice have suggested MMPs and TIMPs as potential candidate genes for CLP, based on expression patterns during palate morphogenesis ${ }^{12}$. If their balance is disrupted, birth defects, such as CLP, can occur. Although essential for the morphogenesis of craniofacial structures, MMPs and TIMPs have not been extensively studied in tissue samples obtained from fully developed children with CLP.

Mouse embryos show distinct temporospatial expression of MMP-2, MMP-3, MMP-7, MMP-9, MMP-13 and TIMP-1, TIMP-2 during palatal fusion. The inactivation of MMPs by TIMPs in vitro leads to a failure of fusion of mouse palatal shelves ${ }^{13}$. It has been reported that two MMP-mediated pathways are involved in palatal fusion, MMP-3 and the MMP-2/a cell membrane-associated MMP (MT1-MMP)/TIMP-2 pathway. Furthermore, a promoter variant in the TIMP-2 gene was associated with NSCLP in Europeans ${ }^{14}$. TIMP-2 is an inhibitor of MMPs and paradoxically it can function as a co-activator of pro-MMP-2 as well ${ }^{15}$. In our study, TIMP-2 positive cell distribution varied from a few to moderate positive structures in the patients examined, and TIMP-2 expression in the oral mucosa was lower in the control group than the CLP group. Particularly, elevated MMP-2 expression was observed in epithelial cells from patients with CBCLP. These results suggest the involvement of the MMP-2/MT-

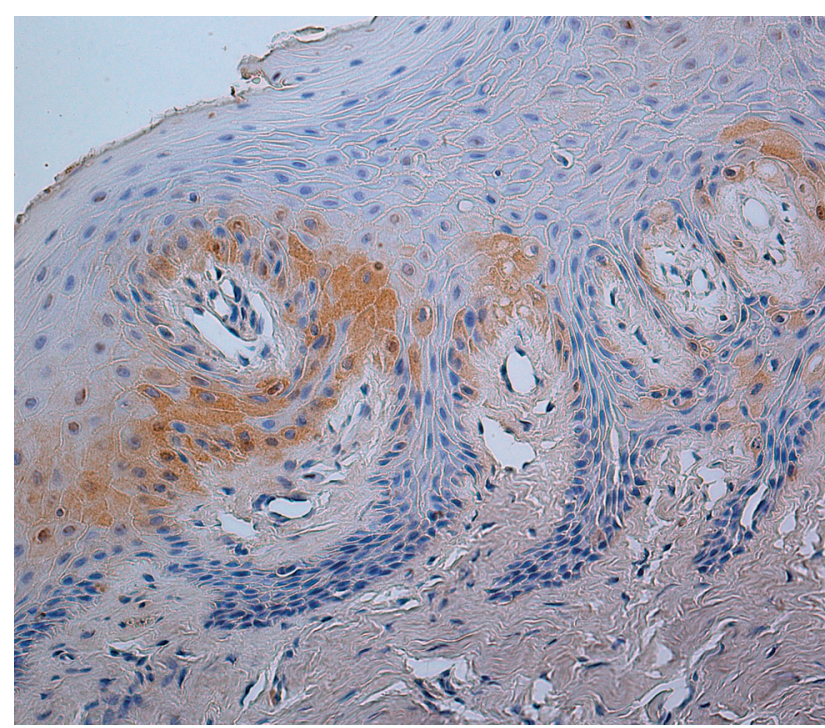

Fig. 6. Few MMP-2-positive epithelial cells in oral epithelial cells in soft tissue from an unaffected 6-years-old child. $200 \mathrm{x}$ magnification.

MMP/TIMP-2 pathway in the ECM remodelling directly associated with CBCLP.

The role of TIMP-4 local expression in humans CLP affected tissue has not been reported.

TIMP-4 may also play a role in activation of proMMP-2. Interestingly, Zhang et al. (2003) suggested that TIMP-4 may indirectly regulate MMP-2 and MMP-9 gene transcription levels ${ }^{16}$. MMP-9 is known for its activity in degrading type IV collagen, the main component of the ECM, and facilitates cell migration ${ }^{17}$. Although Letra et al. (2007) provided no evidence of a major role for this gene in NSCLP (ref. ${ }^{18}$ ), in this study elevated TIMP-4, MMP-9 expression was observed in epithelial cells, fibroblasts, and macrophages from patients with CUCLP and unaffected individuals. In contrast, there were fewer TIMP-4, MMP-9-positive cells in tissue samples from patients with CBCLP. These results show that lower MMP-9 expression might provide a predominantly scarring signal.

MMP-8 (collagenase-2) of the fibrillar collagenase family that also includes MMP-1 and MMP13, is particularly interesting in the context of CLP affected tissue remodelling because it can be expressed by a wide variety of cell types including epithelial cells, fibroblasts, endothelial cells, macrophages and neutrophil leukocytes ${ }^{19}$. It has been shown that MMP-8 is present in multiple embryonic tissues, including neural crest cells ${ }^{20}$. Tissues of the craniofacial complex are primarily derived from neural crest cells. Moreover, MMP-8 is expressed during many pathological conditions associated with excessive degradation of the ECM, such as periodontitis, osteoarthritis, chronic ulcers, and malignant tumours. This study shows that MMP-8-positive cell distribution varied from occasional to numerous positive structures in the patients. The results suggest involvement of MMP-8 locally in the breakdown of ECM in CLP affected tissue. 


\section{CONCLUSIONS}

The results of this study suggest that TIMP-4 and MMP-9 are the main ECM remodeling regulatory proteins expressed in CUCLP affected tissues of the oral mucosa. The increased expression of MMP-2 and TIMP-2 in CLP tissues implicates these factors in the regulation of cell migration during ECM turnover independently of different types of clefts. Investigation of MMP and TIMP expression in tissue samples obtained from patients with CLP is a promising approach to the etiopathogenesis of CLP.

Acknowledgment: The study was funded by RSU project "Longitudinal research on cleft morphopathogenesis". Author contributions: LSF: literature search, manuscript writing, data analysis, statistical analysis; LSF, MP, IA: study design, data collection; LSF, MP: data interpretation.

Conflict of interest statement: The authors state that there are no conflicts of interest regarding the publication of this article.

\section{REFERENCES}

1. Mossey PA, Little J, Munger RG, Dixon MJ, Shaw WC. Cleft lip and palate. Lancet 2009;374(9703):1773-85.

2. Jugessur A, Farlie PG, Kilpatrick N. The genetics of isolated orofacial clefts: from genotypes to subphenotypes. Oral Dis 2009;15(7):43753.

3. Murray JC. Gene/environment causes of cleft lip and/or palate. Clin Genet 2002;61(4):248-56.

4. Dudas M, Li WY, Kim J, Yang A, Kaartinen V. Palatal fusion - where do the midline cells go? A review on cleft palate, a major human birth defect. Acta Histochem 2007;109(1):1-14.

5. Jin JZ, Ding J. Analysis of cell migration, transdifferentiation and apoptosis during mouse secondary palate fusion. Development 2006;133(17):3341-7.

6. Nawshad A. Palatal seam disintegration: to die or not to die? that is no longer the question. Dev Dyn 2008;237(10):2643-56.
7. Mansell JP, Kerrigan J, McGill J, Bailey J, TeKoppele J, Sandy JR. Temporal changes in collagen composition and metabolism during rodent palatogenesis. Mech Ageing Dev 2000;119(1-2):49-62.

8. François-Fiquet C, Poli-Merol ML, Nguyen P, Landais E, Gaillard D Doco-Fenzy M. Role of angiogenesis-related genes in cleft lip/ palate: review of the literature. Int J Pediatr Otorhinolaryngol 2014;78(10):1579-85.

9. Shi J, Son MY, Yamada S, Szabova L, Kahan S, Chrysovergis K, Wolf L, Surmak A, Holmbeck K. Membrane-type MMPs enable extracellular matrix permissiveness and mesenchymal cell proliferation during embryogenesis. Dev Biol 2008;313(1):196-209.

10. Pilmane M, Rumba I, Sundler F. \& Luts A. Patterns of distribution and occurrence of neuroendocrine elements in lungs of humans with chronic lung disease. Proc Latv Acad Sci 1998;52:144-152.

11. Meng L, Bian Z, Torensma R, Von den Hoff JW. Biological mechanisms in palatogenesis and cleft palate. J Dent Res 2009;88(1):22-33.

12. Morris-Wiman J, Burch H, Basco E. Temporospatial distribution of matrix metalloproteinase and tissue inhibitors of matrix metalloproteinases during murine secondary palate morphogenesis. Anat Embryol (Berl) 2000;202(2):129-41.

13. Blavier L, Lazaryev A, Groffen J, Heisterkamp N, DeClerck YA Kaartinen V. TGF-beta3-induced palatogenesis requires matrix metalloproteinases. Mol Biol Cell 2001;12(5):1457-66.

14. Nikopensius $T$, Kempa I, Ambrozaitytè $L$, Jagomägi $T$, Saag $M$, Matulevičienė A, Utkus A, Krjutškov K, Tammekivi V, Piekuse L, Akota I, Barkane B, Krumina A, Klovins J, Lace B, Kučinskas V, Metspalu A. Variation in FGF1, FOXE1, and TIMP2 genes is associated with nonsyndromic cleft lip with or without cleft palate. Birth Defects Res A Clin Mol Teratol 2011;91(4):218-25.

15. Wang $Z$, Juttermann R, Soloway PD. TIMP-2 is required for efficient activation of proMMP-2 in vivo. J Biol Chem 2000;275(34):26411-5.

16. Zhang J, Zhao YG, Cao YJ, Sang OX, Duan EK. Expression and implications of tissue inhibitor of metalloproteinases-4 in mouse embryo. Mol Hum Reprod 2003;9(3):143-9.

17. Zhang B, Henney A, Eriksson P, Hamsten A, Watkins H, Ye S. Genetic variation at the matrix metalloproteinase-9 locus on chromosome 20q12.2-13.1. Hum Genet 1999;105(5):418-23.

18. Letra A, da Silva RA, Menezes R, de Souza AP, de Almeida AL, Sogayar MC, Granjeiro JM. Studies with MMP9 gene promoter polymorphism and nonsyndromic cleft lip and palate. Am J Med Genet A 2007;143A(1):89-91.

19. Van Lint $P$, Libert $C$. Matrix metalloproteinase-8: cleavage can be decisive. Cytokine Growth Factor Rev 2006;17(4):217-23.

20. Giambernardi TA, Sakaguchi AY, Gluhak J, Pavlin D, Troyer DA, Das $\mathrm{G}$, Rodeck U, Klebe RJ. Neutrophil collagenase (MMP-8) is expressed during early development in neural crest cells as well as in adult melanoma cells. Matrix Biol 2001;20(8):577-87. 